\title{
SOIL PROPERTIES OF SHOLA FORESTS INVADED BY BLACK WATTLE (ACACIA MEARNSII) IN THE WESTERN GHATS OF TAMIL NADU, INDIA
}

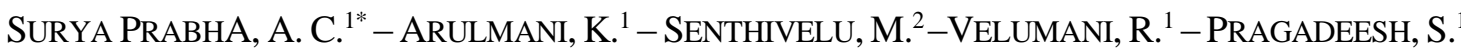 \\ ${ }^{1}$ Silviculture and Forest Management Division, Institute of Forest Genetics and Tree Breeding, \\ Coimbatore, Tamil Nadu 641002, India \\ ${ }^{2}$ Department of Oilseeds, Tamil Nadu Agricultural University, Coimbatore, Tamil Nadu, India \\ *Corresponding author \\ e-mail: acsuryaprabha19@gmail.com; phone: +91-94-8746-8371
}

(Received $20^{\text {th }} \mathrm{Jul} 2021$; accepted $28^{\text {th }}$ Oct 2021)

\begin{abstract}
The Australian Black Wattle (A. mearnsii) threatens native habitats by outcompeting indigenous vegetation for water, soil nutrients and organic matter. The present investigation was conducted to study the soil properties of shola forests invaded by A. mearnsii plantations in the Kodaikanal Forest Division of the Western Ghats of Tamil Nadu, India. Soil samples were collected from A. mearnsii invaded shola forests of Poombarai forest range in the Kodaikanal Forest Division. For comparison purposes, soil samples were collected from shola forests, A. mearnsii plantation, grass land and pine plantations. Soil $\mathrm{pH}(5.02)$, organic carbon $(5.82 \%)$, available nitrogen $\left(201.6 \mathrm{~kg} \mathrm{ha}^{-1}\right)$ and available potassium (334.5 $\left.\mathrm{kg} \mathrm{ha}^{-1}\right)$, exchangeable calcium $(3.79 \mathrm{meq} / 100 \mathrm{~g})$ and exchangeable magnesium $(5.18 \mathrm{meq} / 100 \mathrm{~g})$ were highest under A. mearnsii invaded shola forest at 0-30 $\mathrm{cm}$ depth. Available $\mathrm{P}$ was highest under A. mearnsii plantation $\left(21.5 \mathrm{~kg} \mathrm{ha}^{-1}\right)$. The control plot (shola forest), registered a $\mathrm{pH}$ of 3.94 , organic carbon $(5.80 \%)$, available nitrogen $\left(180.4 \mathrm{~kg} \mathrm{ha}^{-1}\right)$, available phosphorus $\left(21.0 \mathrm{~kg} \mathrm{ha}^{-1}\right)$, available potassium $\left(318.3 \mathrm{~kg} \mathrm{ha}^{-1}\right)$, exchangeable calcium $(3.59 \mathrm{meq} / 100 \mathrm{~g})$ and exchangeable magnesium $(5.08 \mathrm{meq} / 100 \mathrm{~g})$. The baseline data generated from the study can be utilized for undertaking appropriate decision making in the management of forests to control the invasion menace of A. mearnsii thereby improving the soil quality.
\end{abstract}

Keywords: invasion ecology, habitat modification, invasive species, soil characteristics

\section{Introduction}

A collective of Tropical Montane, evergreen forests locally known as sholas is situated in the higher mountain tracts of the Western Ghats, at an altitude above $1500 \mathrm{~m}$ interspersed with rolling grasslands. Shola forests have high ecological significance in protecting the headwaters of rivers by holding back water received by precipitation like a sponge, thus preventing rapid run-off. These diverse forests and grasslands were converted into commercially valuable plantations to meet the fuel wood requirement of tea industry during British period. Some of the introduced species like A. mearnsii have become invasive alien species in this ecosystem and became a serious threat to this high-altitude ecosystem (Myers et al., 2000).

The Australian Black Wattle (A. mearnsii) was introduced during the 1960s in State Forest lands located in the upper altitudes of the Palani hills- an eastern offshoot of the Western Ghats, a mountain range that runs parallel to the southwest coast of Peninsular India near the hill station of Kodaikanal (Mitchell, 1972; Mathew et al., 1975). A. mearnsii, a small to large, evergreen, single stemmed or multi branched tree threatens native habitats by outcompeting indigenous vegetation for water, soil nutrients and organic matter (Moyo and Fatunbi, 2010). Invasive alien plants alter the invaded 
community by shifting native biodiversity through a reduction in light penetration, changes in soil nutrients and hydrology and then modifying ecosystem functionality (Vardien et al., 2012). There are various mechanisms whereby invasive alien plants can alter soil ecosystems (Tererai et al., 2015). Studies have reported the mass of the leaf litter in areas invaded by $A$. mearnsii to be greater than that of un-invaded areas, thereby the dense layer inhibits the establishment of native seedlings (Pandey et al., 2014). Between 1849 and 1992, in the Nilgiris Biosphere Reserve, the sholas decreased from 8600 ha to 4225 ha and grasslands from 29875 ha to 4700 ha (Kumar, 1993).

Soil is the major source of nutrients for the growth of plants and determining the degree of soil physico-chemical characteristics are very necessary to evaluate the soil fertility. The nutrient transformation and its availability in soils depend on $\mathrm{pH}$, clay minerals, cation and anion exchange capacity (Reddy and Reddy, 2010). Soils exhibit difference in properties in relation to the vegetation changes and vary spatially primarily in response to rooting and litter fall characteristics of the perennial vegetation on more or less the same soil material (Balagopalan et al., 1993). Invasive plant species can modify physical or chemical attributes of soil, including inputs and cycling of nitrogen and other elements (Ehrenfeld, 2003; Haubensaket al., 2004 and Hawkes et al., 2005; Tererai et al., 2015). The amount of soil nutrients especially soil nitrogen, phosphorus and carbon, soil $\mathrm{pH}$, microbial $\mathrm{N}$ and $\mathrm{P}$, decomposition rates and soil water repellents can be increased due to invasive alien plants compared to the natural site (Fan et al., 2010; Ruwanza et al., 2013; Ruwanza and Shackleton, 2016). However, in some studies, they may show the reverse trends (Ehrenfeld, 2003; Tererai et al., 2015). Nutrient dynamics may also become altered as a result of changes in the physical properties of the soil caused by the introduction of new species.

Studies on the properties of soil in invaded shola forests are required for proper management of the forests and utilization of resources. Studies on the assessment of soil properties under shola forests invaded by $A$. mearnsii in the Western Ghats of Kodaikanal are scanty. Therefore, the present study was proposed with an aim to study the soil properties of shola forests invaded by A. mearnsii plantations in the Western Ghats of Kodaikanal, Tamil Nadu, India.

\section{Materials and methods}

\section{Location and site description}

The present study was carried out in the Western Ghats of Tamil Nadu covering the Poombarai Range of the Kodaikanal Forest Division, Tamil Nadu, India (Fig. 1). Kodaikanal Forest Division lies within $10^{\circ} 6^{\prime}$ and $10^{\circ} 21^{\prime}$ North latitudes and $77^{\circ} 16^{\prime}$ and $77^{\circ} 42^{\prime}$ 'East longitudes and is surrounded by Kerala state in West, Indira Gandhi Wild life sanctuary, Pollachi in North-west, Dindigul forest division on the North-east and Theni revenue district in South. The altitude varies from 300 to $2654 \mathrm{~m}$ and this area experiences an average yearly rainfall of $165 \mathrm{~cm}$. The average annual temperature in Kodaikanal $20.8^{\circ} \mathrm{C}$.

\section{Soil sampling and analysis}

Soil samples were collected from the different study plots viz, shola forests invaded by $A$. mearnsii, shola forests, A. mearnsii plantation, grass land and pine plantations during 2016-2018 (Fig. 2). At each of the study plot, soil samples were collected from three sites and at four depths viz., 0-30, 30-50, 50-80 and 80-100 cm. Sites within each 
invasion treatment were approximately $200 \mathrm{~m}$ apart to provide a measure of independence (Galatowitsch and Richardson, 2005).

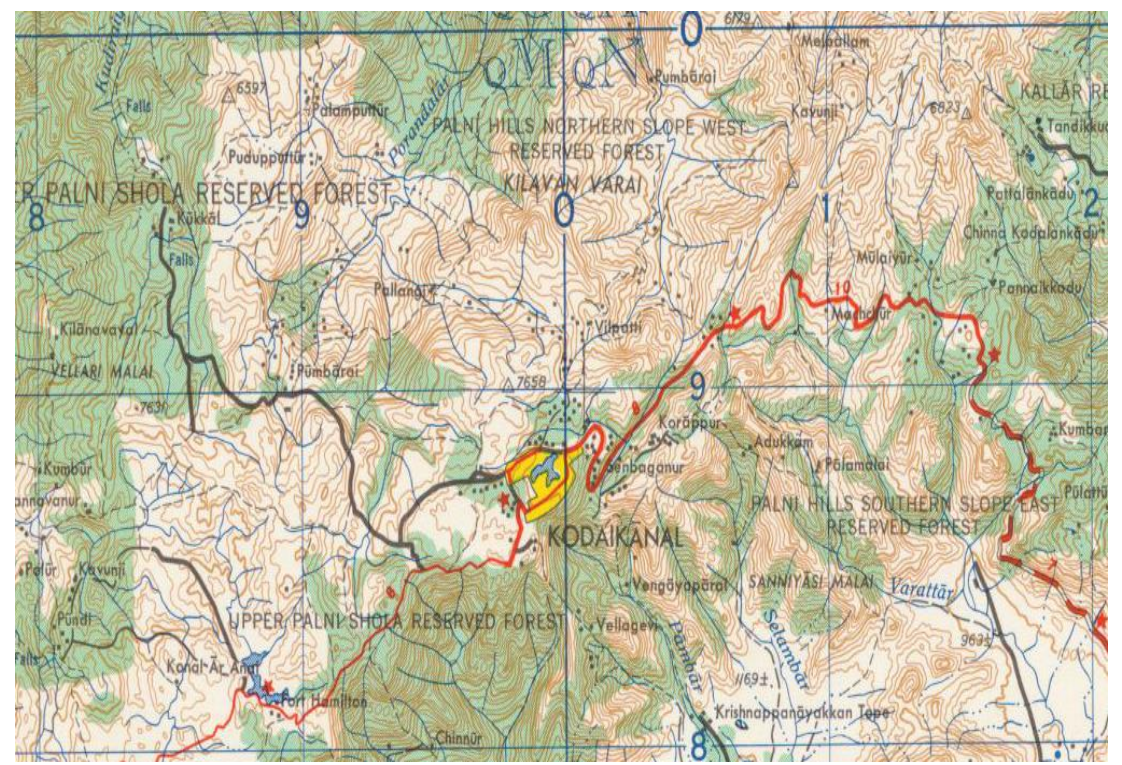

Figure 1. Map showing the study area

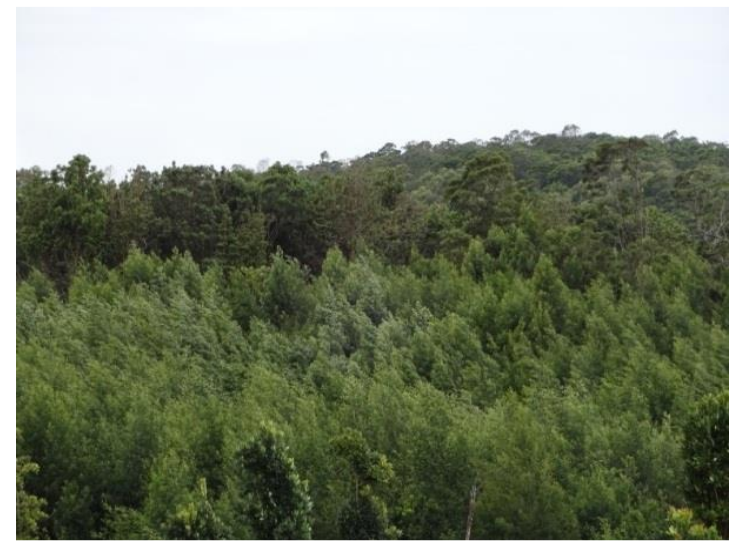

(a)

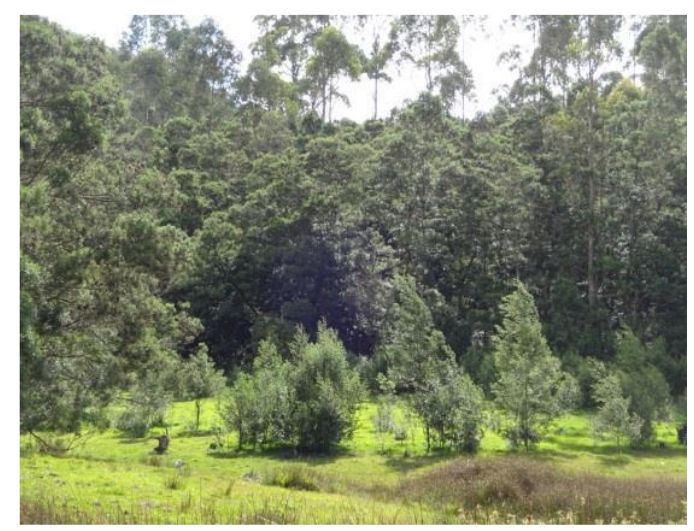

(c)

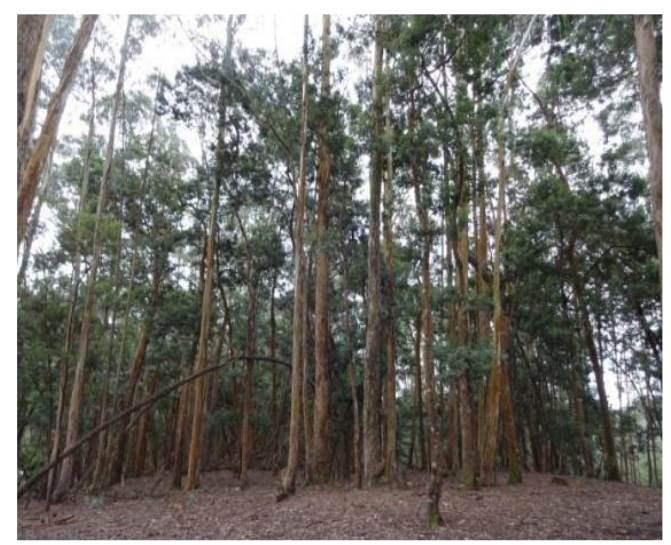

(b)

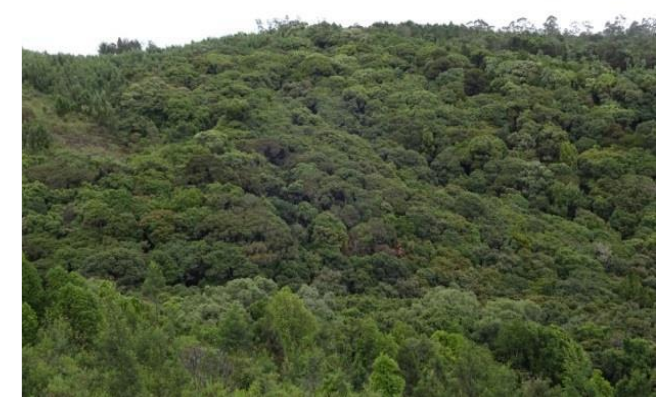

APPLIED ECOLOGY AND ENVIRONMENTAL RESEARCH 20(1):251-263.

http://www.aloki.hu • ISSN 15891623 (Print) • ISSN 17850037 (Online)

DOI: http://dx.doi.org/10.15666/aeer/2001_251263

(c) 2022, ALÖKI Kft., Budapest, Hungary 

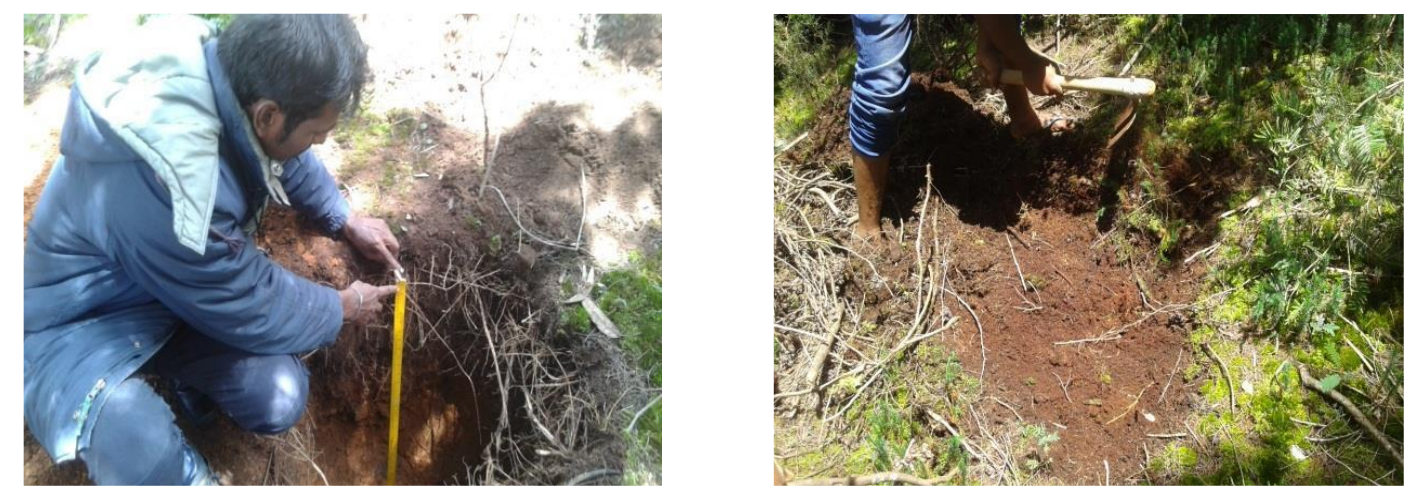

(d)

Figure 2. (a) Acacia mearnsii invaded shola forests. (b) Acacia mearnsii plantation. (c) A. mearnsii invaded grassland; Evergreen shola forest (d) Soil sampling in Acacia mearnsii invaded sites

At each sampling point, an area of $0.5 \mathrm{~m} \times 0.5 \mathrm{~m}$ was removed and a pit of $50 \mathrm{~cm}$ wide, 50 $\mathrm{cm}$ in length and $100 \mathrm{~cm}$ deep was dug. The soil was scrapped from three sides of the pit with the help of a kurpee at each depth. The soil was mixed thoroughly and transferred to a polythene bag with proper labeling. The samples were air-dried and sieved to pass through 2 $\mathrm{mm}$ mesh sieve for physico-chemical analysis. The $\mathrm{pH}$ of soil was determined using an aqueous suspension of soil (soil and water in 1:2.5 ratio) using a digital micro controller based pH meter with electrode, Make: Systronics (Jackson, 1973). Soil organic carbon was estimated by standard Chromic acid wet oxidation method (Walkley and Black, 1934). The available nitrogen in the soil was estimated by the alkaline permanganate method using Automatic Nitrogen Analyzer, Make: Pelican (Subbiah and Asija, 1956).

The concentration of available phosphorus was determined by Olsen, Bray 1 and Kurtz extraction methods using UV-Vis Spectrophotometer, Make: Shimadzu (Olsen et al., 1954; Bray and Kurtz, 1945). Available potassium was estimated by neutral normal ammonium acetate extraction using Flame Photometer, Make: Systronics (Stanford and English, 1949). The contents of exchangeable bases $(\mathrm{Ca}$ and $\mathrm{Mg}$,) were determined by Versenate titration after extraction using $1 \mathrm{~N}$ ammonium acetate adjusted to $\mathrm{pH} 7.0$ (Cheng and Bray, 1951). Available micronutrients viz, $\mathrm{Fe}, \mathrm{Mn}, \mathrm{Zn}$ and $\mathrm{Cu}$ were extracted using Diethylene Triamine Penta Acetic acid reagent and the micronutrients content were estimated using Atomic Absorption Spectrophotometer, Make: Shimadzu (Lindsay and Norvell, 1978).

\section{Statistical analysis}

The data obtained were subjected to statistical analysis in SPSS ${ }^{\circledR} 19.0$ version statistical software. One-way ANOVA was also applied to analyse and to compare the mean significant difference between each parameter under different sites. During the ANOVA test the soil properties were the dependent variables while sampling sites were the independent variables.

\section{Results}

\section{Acacia mearnsii invasion on soil properties}

Analysis of variance (ANOVA) testing the effects of A. mearnsii on soil properties among the study plots of Poombarai forest range at 0 to $30 \mathrm{~cm}$ depth is given in Table 1 . The soils in all the sites were acidic in general and soil $\mathrm{pH}$ was highest under $A$. 
mearnsii invaded shola forest $(\mathrm{p}<0.001)$ ) at $0-30 \mathrm{~cm}$ depth and was followed by grassland. Shola forest registered the lowest $\mathrm{pH}$ (3.94) at 0-30 cm depth. At $30-50 \mathrm{~cm}$, $50-80 \mathrm{~cm}$ and $80-100 \mathrm{~cm}$, soil $\mathrm{pH}$ was highest under A. mearnsii invaded shola forest at (Tables 2, 3 and 4).

Table 1. Analysis of variance (ANOVA) testing the effects of Acacia mearnsii on soil properties among the study plots of Poombarai forest range at 0 to $30 \mathrm{~cm}$ depth

\begin{tabular}{|c|c|c|c|c|c|c|c|}
\hline \multirow[b]{2}{*}{ Soil properties } & \multicolumn{5}{|c|}{ Study plots } & \multirow[b]{2}{*}{ F-value } & \multirow[b]{2}{*}{ p-value } \\
\hline & $\begin{array}{c}\text { A. mearnsii } \\
\text { invaded shola }\end{array}$ & $\begin{array}{l}\text { A. mearnsii } \\
\text { plantation }\end{array}$ & Grassland & Pine forest & Shola forest & & \\
\hline Soil pH & $5.02 \pm 0.03$ & $4.13 \pm 0.02$ & $4.39 \pm 0.02$ & $4.15 \pm 0.02$ & $3.94 \pm 0.02$ & 296.68 & $<0.001$ \\
\hline $\begin{array}{l}\text { Electrical conductivity } \\
\left(\mathrm{dS} \mathrm{m}^{-1}\right)\end{array}$ & $0.42 \pm 0.03$ & $0.62 \pm 0.06$ & $0.41 \pm 0.03$ & $0.30 \pm 0.03$ & $0.36 \pm 0.03$ & 954.78 & $<0.001$ \\
\hline Organic carbon $(\%)$ & $5.82 \pm 0.03$ & $3.97 \pm 0.02$ & $4.3 \pm 0.02$ & $5.54 \pm 0.03$ & $5.8 \pm 0.03$ & 835.15 & $<0.001$ \\
\hline $\begin{array}{c}\text { Available nitrogen } \\
\left(\mathrm{kg} \mathrm{ha}^{-1}\right)\end{array}$ & $201.6 \pm 1.16$ & $168.6 \pm 0.97$ & $164 \pm 0.95$ & $145.6 \pm 0.84$ & $180.4 \pm 1.04$ & 429.87 & $<0.001$ \\
\hline $\begin{array}{c}\text { Available phosphorus } \\
\left(\mathrm{kg} \mathrm{h}^{-1}\right)\end{array}$ & $19.9 \pm 0.11$ & $21.5 \pm 0.12$ & $20.6 \pm 0.12$ & $20.1 \pm 0.11$ & $21.0 \pm 0.12$ & 29.97 & $<0.001$ \\
\hline $\begin{array}{l}\text { Available potassium } \\
\left(\mathrm{kg} \mathrm{ha}^{-1}\right)\end{array}$ & $334.5 \pm 1.93$ & $183.6 \pm 1.06$ & $164.8 \pm 0.95$ & $139.2 \pm 0.80$ & $318.3 \pm 1.84$ & 4262.04 & $<0.001$ \\
\hline $\begin{array}{l}\text { Exchangeable calcium } \\
(\text { meq } / 100 \mathrm{~g})\end{array}$ & $3.79 \pm 0.04$ & $1.99 \pm 0.02$ & $3.59 \pm 0.03$ & $3.39 \pm 0.02$ & $3.59 \pm 0.03$ & 587.56 & $<0.001$ \\
\hline $\begin{array}{c}\text { Exchangeable } \\
\text { magnesium }(\mathrm{meq} / 100 \mathrm{~g})\end{array}$ & $5.18 \pm 0.04$ & $4.78 \pm 0.04$ & $4.09 \pm 0.03$ & $4.68 \pm 0.04$ & $5.08 \pm 0.04$ & 106.06 & $<0.001$ \\
\hline DTPA-Cu (ppm) & $2.99 \pm 0.03$ & $1.2 \pm 0.01$ & $2.79 \pm 0.03$ & $0.6 \pm 0.01$ & $5.58 \pm 0.05$ & 4717.02 & $<0.001$ \\
\hline DTPA-Zn (ppm) & $1.00 \pm 0.01$ & $0.5 \pm 0.01$ & $0.8 \pm 0.01$ & $0.3 \pm 0.0$ & $1.2 \pm 0.01$ & 2386.24 & $<0.001$ \\
\hline DTPA-Mn (ppm) & $2.4 \pm 0.01$ & $0.6 \pm 0.01$ & $0.4 \pm 0.0$ & $0.2 \pm 0.0$ & $2.6 \pm 0.02$ & 14442.85 & $<0.001$ \\
\hline DTPA-Fe (ppm) & $10.4 \pm 0.06$ & $7.6 \pm 0.05$ & $6.4 \pm 0.03$ & $5.9 \pm 0.03$ & $20.1 \pm 0.11$ & 8117.2 & $<0.001$ \\
\hline
\end{tabular}

Table 2. Analysis of variance (ANOVA) testing the effects of Acacia mearnsii on soil properties among the study plots of Poombarai forest range at 30 to $50 \mathrm{~cm}$ depth

\begin{tabular}{|c|c|c|c|c|c|c|c|}
\hline \multirow[b]{2}{*}{ Soil properties } & \multicolumn{5}{|c|}{ Study plots } & \multirow[b]{2}{*}{ F-value } & \multirow[b]{2}{*}{ p-value } \\
\hline & $\begin{array}{c}\text { A. mearnsii } \\
\text { invaded shola }\end{array}$ & $\begin{array}{l}\text { A. mearnsii } \\
\text { plantation }\end{array}$ & Grassland & Pine forest & Shola forest & & \\
\hline Soil pH & $5.04 \pm 0.03$ & $4.23 \pm 0.02$ & $4.56 \pm 0.02$ & $4.23 \pm 0.02$ & $3.95 \pm 0.02$ & 266.12 & $<0.001$ \\
\hline $\begin{array}{c}\text { Electrical conductivity } \\
\left(\mathrm{dS} \mathrm{m}^{-1}\right)\end{array}$ & $0.4 \pm 0.00$ & $0.54 \pm 0.06$ & $0.38 \pm 0.00$ & $0.28 \pm 0.00$ & $0.38 \pm 0.00$ & 1302.0 & $<0.001$ \\
\hline Organic carbon $(\%)$ & $5.11 \pm 0.03$ & $3.89 \pm 0.02$ & $4.25 \pm 0.02$ & $4.87 \pm 0.03$ & $5.6 \pm 0.03$ & 589.80 & $<0.001$ \\
\hline $\begin{array}{l}\text { Available nitrogen } \\
\quad\left(\mathrm{kg} \mathrm{ha}^{-1}\right)\end{array}$ & $168 \pm 0.97$ & $154.2 \pm 0.88$ & $150.2 \pm 0.87$ & $124.2 \pm 0.72$ & $164.8 \pm 0.95$ & 384.59 & $<0.001$ \\
\hline $\begin{array}{c}\text { Available phosphorus } \\
\left(\mathrm{kg} \mathrm{ha}^{-1}\right)\end{array}$ & $19.7 \pm 0.11$ & $20.8 \pm 0.12$ & $20.4 \pm 0.11$ & $20.3 \pm 0.11$ & $20.6 \pm 0.12$ & 12.46 & $<0.001$ \\
\hline $\begin{array}{c}\text { Available potassium } \\
\left(\mathrm{kg} \mathrm{ha}^{-1}\right)\end{array}$ & $326.3 \pm 1.88$ & $152.8 \pm 0.88$ & $152.8 \pm 0.88$ & $130.2 \pm 0.75$ & $302.6 \pm 1.75$ & 5008.86 & $<0.001$ \\
\hline $\begin{array}{l}\text { Exchangeable calcium } \\
(\mathrm{meq} / 100 \mathrm{~g})\end{array}$ & $3.2 \pm 0.02$ & $2.0 \pm 0.01$ & $3.2 \pm 0.02$ & $2.8 \pm 0.02$ & $3.4 \pm 0.02$ & 1170.0 & $<0.001$ \\
\hline $\begin{array}{c}\text { Exchangeable } \\
\text { magnesium }(\mathrm{meq} / 100 \mathrm{~g})\end{array}$ & $4.8 \pm 0.03$ & $4.5 \pm 0.02$ & $3.6 \pm 0.02$ & $4.6 \pm 0.03$ & $4.7 \pm 0.03$ & 314.06 & $<0.001$ \\
\hline DTPA-Cu (ppm) & $2.6 \pm 0.02$ & $1.0 \pm 0.01$ & $2.6 \pm 0.02$ & $0.4 \pm 0.0$ & $4.5 \pm 0.03$ & 9822.97 & $<0.001$ \\
\hline DTPA-Zn (ppm) & $0.6 \pm 0.01$ & $0.3 \pm 0.0$ & $0.6 \pm 0.01$ & $0.2 \pm 0.0$ & $1.0 \pm 0.01$ & 4900.00 & $<0.001$ \\
\hline DTPA-Mn (ppm) & $2.0 \pm 0.01$ & $0.4 \pm 0.0$ & $0.2 \pm 0.0$ & $0.1 \pm 0.0$ & $2.0 \pm 0.01$ & 17775.00 & $<0.001$ \\
\hline DTPA-Fe (ppm) & $8.2 \pm 0.05$ & $6.7 \pm 0.04$ & $6.2 \pm 0.03$ & $4.6 \pm 0.03$ & $14.6 \pm 0.09$ & 5645.86 & $<0.001$ \\
\hline
\end{tabular}


Table 3. Analysis of variance (ANOVA) testing the effects of Acacia mearnsii on soil properties among the study plots of Poombarai forest range at 50 to $80 \mathrm{~cm}$ depth

\begin{tabular}{|c|c|c|c|c|c|c|c|}
\hline \multirow[b]{2}{*}{ Soil properties } & \multicolumn{5}{|c|}{ Study plots } & \multirow[b]{2}{*}{ F-value } & \multirow[b]{2}{*}{ p-value } \\
\hline & $\begin{array}{c}\text { A. mearnsii } \\
\text { invaded shola }\end{array}$ & $\begin{array}{c}\text { A. mearnsii } \\
\text { plantation }\end{array}$ & Grassland & Pine forest & Shola forest & & \\
\hline Soil pH & $5.32 \pm 0.03$ & $4.35 \pm 0.02$ & $4.78 \pm 0.03$ & $4.85 \pm 0.03$ & $3.88 \pm 0.02$ & 416.25 & $<0.001$ \\
\hline $\begin{array}{l}\text { Electrical conductivity } \\
\left(\mathrm{dS} \mathrm{m}^{-1}\right)\end{array}$ & $0.34 \pm 0.00$ & $0.58 \pm 0.01$ & $0.49 \pm 0.00$ & $0.20 \pm 0.00$ & $0.21 \pm 0.00$ & 4264.5 & $<0.001$ \\
\hline Organic carbon $(\%)$ & $4.25 \pm 0.02$ & $3.42 \pm 0.01$ & $4.02 \pm 0.02$ & $4.56 \pm 0.03$ & $5.23 \pm 0.03$ & 736.69 & $<0.001$ \\
\hline $\begin{array}{l}\text { Available nitrogen } \\
\quad\left(\mathrm{kg} \mathrm{ha}^{-1}\right)\end{array}$ & $156.8 \pm 0.90$ & $150.6 \pm 0.87$ & $126.4 \pm 0.73$ & $118.6 \pm 0.68$ & $152.4 \pm 0.88$ & 442.38 & $<0.001$ \\
\hline $\begin{array}{l}\text { Available phosphorus } \\
\left(\mathrm{kg} \mathrm{ha}^{-1}\right)\end{array}$ & $18.2 \pm 0.10$ & $20.4 \pm 0.11$ & $18.2 \pm 0.10$ & $19.3 \pm 0.11$ & $19.4 \pm 0.11$ & 72.88 & $<0.001$ \\
\hline $\begin{array}{l}\text { Available potassium } \\
\left(\mathrm{kg} \mathrm{ha}^{-1}\right)\end{array}$ & $276.5 \pm 1.59$ & $145.2 \pm 0.84$ & $146.7 \pm 0.85$ & $126.4 \pm 0.72$ & $286.4 \pm 1.65$ & 4239.96 & $<0.001$ \\
\hline $\begin{array}{l}\text { Exchangeable calcium } \\
\text { (meq/100 g) }\end{array}$ & $2.8 \pm 0.02$ & $1.2 \pm 0.01$ & $2.4 \pm 0.01$ & $2.6 \pm 0.02$ & $2.7 \pm 0.02$ & 2006.2 & $<0.001$ \\
\hline $\begin{array}{c}\text { Exchangeable magnesium } \\
(\mathrm{meq} / 100 \mathrm{~g})\end{array}$ & $4.6 \pm 0.03$ & $3.8 \pm 0.02$ & $3.2 \pm 0.01$ & $4.1 \pm 0.02$ & $4.5 \pm 0.03$ & 562.53 & $<0.001$ \\
\hline DTPA-Cu (ppm) & $2.2 \pm 0.01$ & $0.6 \pm 0.01$ & $1.9 \pm 0.01$ & $0.2 \pm 0.0$ & $3.4 \pm 0.02$ & 13816.6 & $<0.001$ \\
\hline DTPA-Zn (ppm) & $0.57 \pm 0.01$ & $0.2 \pm 0.0$ & $0.3 \pm 0.0$ & $0.1 \pm 0.0$ & $0.84 \pm 0.01$ & 6796.5 & $<0.001$ \\
\hline DTPA-Mn (ppm) & $1.4 \pm 0.01$ & $0.4 \pm 0.0$ & $0.3 \pm 0.0$ & $0.1 \pm 0.0$ & $1.8 \pm 0.01$ & 16950.00 & $<0.001$ \\
\hline DTPA-Fe (ppm) & $6.8 \pm 0.04$ & $4.3 \pm 0.02$ & $5.8 \pm 0.03$ & $4.2 \pm 0.02$ & $10.2 \pm 0.06$ & 4116.82 & $<0.001$ \\
\hline
\end{tabular}

Table 4. Analysis of variance (ANOVA) testing the effects of Acacia mearnsii on soil properties among the study plots of Poombarai forest range at 80 to $100 \mathrm{~cm}$ depth

\begin{tabular}{|c|c|c|c|c|c|c|c|}
\hline \multirow[b]{2}{*}{ Soil properties } & \multicolumn{5}{|c|}{ Study plots } & \multirow[t]{2}{*}{ F-value } & \multirow[t]{2}{*}{ p-value } \\
\hline & $\begin{array}{c}\text { A. mearnsii } \\
\text { invaded shola }\end{array}$ & $\begin{array}{c}\text { A. mearnsii } \\
\text { plantation }\end{array}$ & Grassland & Pine forest & Shola forest & & \\
\hline Soil pH & $5.28 \pm 0.03$ & $4.38 \pm 0.02$ & $4.69 \pm 0.03$ & $4.73 \pm 0.03$ & $3.86 \pm 0.02$ & 379.44 & $<0.001$ \\
\hline $\begin{array}{l}\text { Electrical conductivity } \\
\left(\mathrm{dS} \mathrm{m}^{-1}\right)\end{array}$ & $0.36 \pm 0.00$ & $0.41 \pm 0.00$ & $0.32 \pm 0.00$ & $0.24 \pm 0.00$ & $0.14 \pm 0.00$ & - & - \\
\hline Organic carbon $(\%)$ & $3.41 \pm 0.02$ & $2.84 \pm 0.02$ & $3.64 \pm 0.02$ & $3.92 \pm 0.02$ & $4.86 \pm 0.03$ & 1107.7 & $<0.001$ \\
\hline $\begin{array}{l}\text { Available nitrogen } \\
\left(\mathrm{kg} \mathrm{ha}^{-1}\right)\end{array}$ & $134.4 \pm 0.77$ & $140.7 \pm 0.81$ & $116.4 \pm 0.67$ & $104.8 \pm 0.61$ & $130.6 \pm 0.76$ & 399.86 & $<0.001$ \\
\hline $\begin{array}{l}\text { Available phosphorus } \\
\qquad\left(\mathrm{kg} \mathrm{ha}^{-1}\right)\end{array}$ & $19.0 \pm 0.11$ & $18.6 \pm 0.10$ & $18.0 \pm 0.10$ & $19.4 \pm 0.11$ & $18.8 \pm 0.11$ & 22.74 & $<0.001$ \\
\hline $\begin{array}{c}\text { Available potassium } \\
\left(\mathrm{kg} \mathrm{ha}^{-1}\right)\end{array}$ & $240.8 \pm 1.39$ & $133.4 \pm 0.77$ & $130.2 \pm 0.75$ & $118.6 \pm 0.68$ & $274.6 \pm 1.59$ & 4329.71 & $<0.001$ \\
\hline $\begin{array}{c}\text { Exchangeable calcium } \\
\text { (meq/100 g) }\end{array}$ & $2.0 \pm 0.01$ & $1.0 \pm 0.01$ & $1.9 \pm 0.01$ & $2.2 \pm 0.01$ & $2.3 \pm 0.01$ & 2355.88 & $<0.001$ \\
\hline $\begin{array}{c}\text { Exchangeable magnesium } \\
(\mathrm{meq} / 100 \mathrm{~g})\end{array}$ & $3.7 \pm 0.02$ & $3.4 \pm 0.01$ & $2.9 \pm 0.01$ & $3.8 \pm 0.02$ & $4.0 \pm 0.02$ & 415.90 & $<0.001$ \\
\hline DTPA-Cu (ppm) & $1.6 \pm 0.01$ & $0.4 \pm 0.0$ & $2.0 \pm 0.01$ & $0.1 \pm 0.0$ & $4.0 \pm 0.02$ & 15012.5 & $<0.001$ \\
\hline DTPA-Zn (ppm) & $0.4 \pm 0.01$ & $0.2 \pm 0.0$ & $0.1 \pm 0.0$ & $0.1 \pm 0.01$ & $0.62 \pm 0.01$ & 7542.0 & $<0.001$ \\
\hline DTPA-Mn (ppm) & $1.8 \pm 0.01$ & $0.2 \pm 0.0$ & $0.1 \pm 0.0$ & $0.2 \pm 0.0$ & $1.2 \pm 0.01$ & 17400.00 & $<0.001$ \\
\hline DTPA-Fe (ppm) & $5.7 \pm 0.03$ & $2.9 \pm 0.02$ & $5.0 \pm 0.03$ & $3.1 \pm 0.02$ & $8.4 \pm 0.05$ & 5262.58 & $<0.001$ \\
\hline
\end{tabular}

Soil organic carbon (SOC) content in the Poombarai range was significantly influenced by A. mearnsii invasion (Fig. 3). SOC content in the study plots varied significantly ( $\mathrm{p}<0.001 \%$ by ANOVA) at different soil depths. Soil organic carbon was highest under A. mearnsii invaded shola forest (5.82\%) and was on par with shola forest 
$(5.80 \%)$ at $0-30 \mathrm{~cm}$ depth. This was followed by pine forest $(5.54 \%)$, and grassland (4.30\%). The lowest SOC was recorded in A. mearnsii plantation (3.97\%).

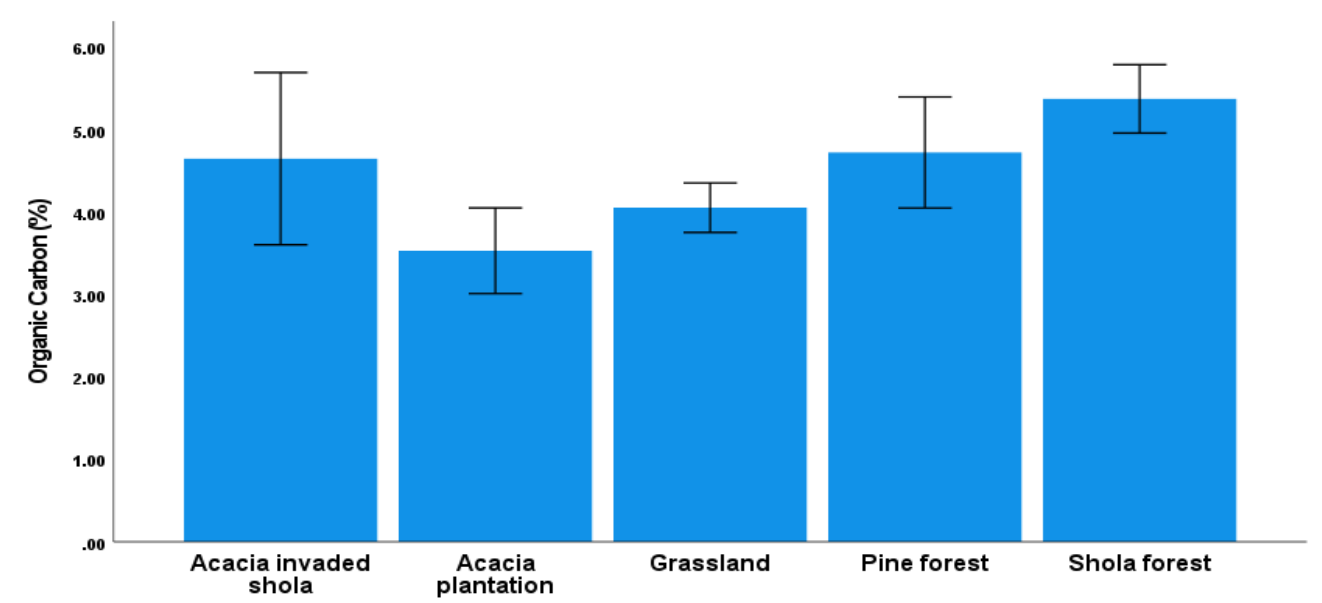

Figure 3. A. mearnsii invasion on soil organic carbon (\%) in Poombarai range

The available nitrogen $\left(\mathrm{kg} \mathrm{ha}^{-1}\right)$ in the different study plots of Poombarai range is presented in Figure 4. At different soil depths, the available nitrogen varied significantly ( $\mathrm{p}<0.001 \%$ by ANOVA) among the study plots. A. mearnsii invaded shola forest recorded the highest available nitrogen $(\mathrm{p}<0.001 \%)$ at $0-30 \mathrm{~cm}$ depth (201.6 kg ha $\mathrm{kg}^{-1}$ ) and was followed by shola forest (180.4 kg ha-1), and A. mearnsii plantation $\left(168.6 \mathrm{~kg} \mathrm{ha}^{-1}\right)$. The lowest available nitrogen was recorded in pine forest (145.6 $\mathrm{kg} \mathrm{ha}^{-1}$ ) at $0-30 \mathrm{~cm}$ depth (Table 1). With respect to soil depth, maximum available nitrogen was registered at $0-30 \mathrm{~cm}$ depth. The available nitrogen showed a decreasing trend with soil depth in all the study plots of Poombarai range. At $30-50 \mathrm{~cm}$, $50-80 \mathrm{~cm}$ and $80-100 \mathrm{~cm}$ nitrogen availability was highest under A. mearnsii invaded shola forest $(\mathrm{p}<0.001 \%)$.

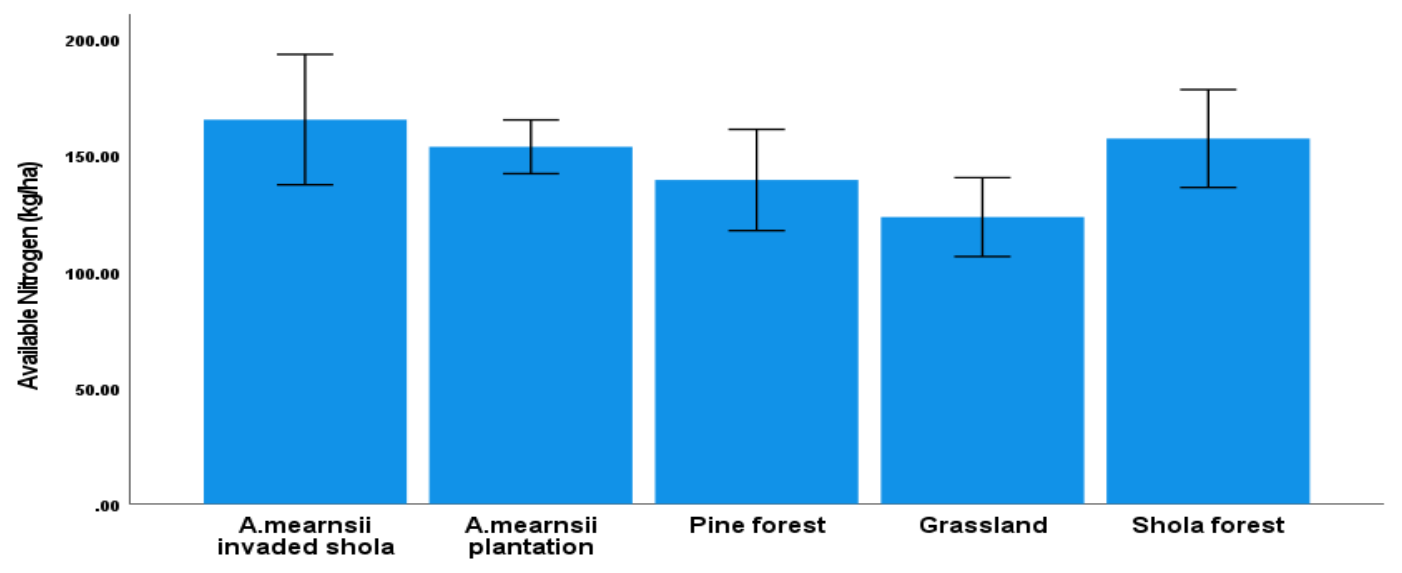

Figure 4. A. Mearnsii invasion on available nitrogen $\left(\mathrm{kg} \mathrm{ha}^{-1}\right)$ in Poombarai range

Available phosphorus was highest under A. mearnsii plantation $\left(21.5 \mathrm{~kg} \mathrm{ha}^{-1}\right)$ at 0 $30 \mathrm{~cm}$ depth. This was on par with shola forest $\left(21.0 \mathrm{~kg} \mathrm{ha}^{-1}\right)$ and was followed by pine 
forest, and grassland (Table 1). Available phosphorus was lowest in A. mearnsii invaded shola forest $\left(19.9 \mathrm{~kg} \mathrm{ha}^{-1}\right)$. At $30-50 \mathrm{~cm}$ and $50-80 \mathrm{~cm}$, A. mearnsii plantation recorded the highest Available Phosphorus $(\mathrm{p}<0.001)$ and was on par with shola forest (Table 2 and 3). Pine forest registered greater available $\mathrm{P}(<0.001)$ at $80-100 \mathrm{~cm}($ Table 4$)$. The available potassium $\left(\mathrm{kg} \mathrm{ha}^{-1}\right)$ in the different study plots of Poombarai range is presented in (Fig. 5). Available potassium varied significantly ( $<<0.001 \%)$ among the study plots. A. mearnsii invaded shola forest recorded the highest available potassium $\left(334.5 \mathrm{~kg} \mathrm{ha}^{-1}\right)$ at $0-30 \mathrm{~cm}$ depth and was followed by shola forest $\left(318.3 \mathrm{~kg} \mathrm{ha}^{-1}\right)$. The lowest available potassium was recorded in pine forest $\left(139.2 \mathrm{~kg} \mathrm{ha}^{-1}\right)$ at $0-30 \mathrm{~cm}$ depth. With respect to soil depth, maximum available potassium was registered at 0-30 $\mathrm{cm}$ depth. The available potassium showed a decreasing trend with soil depth in all the study plots (Table 1).

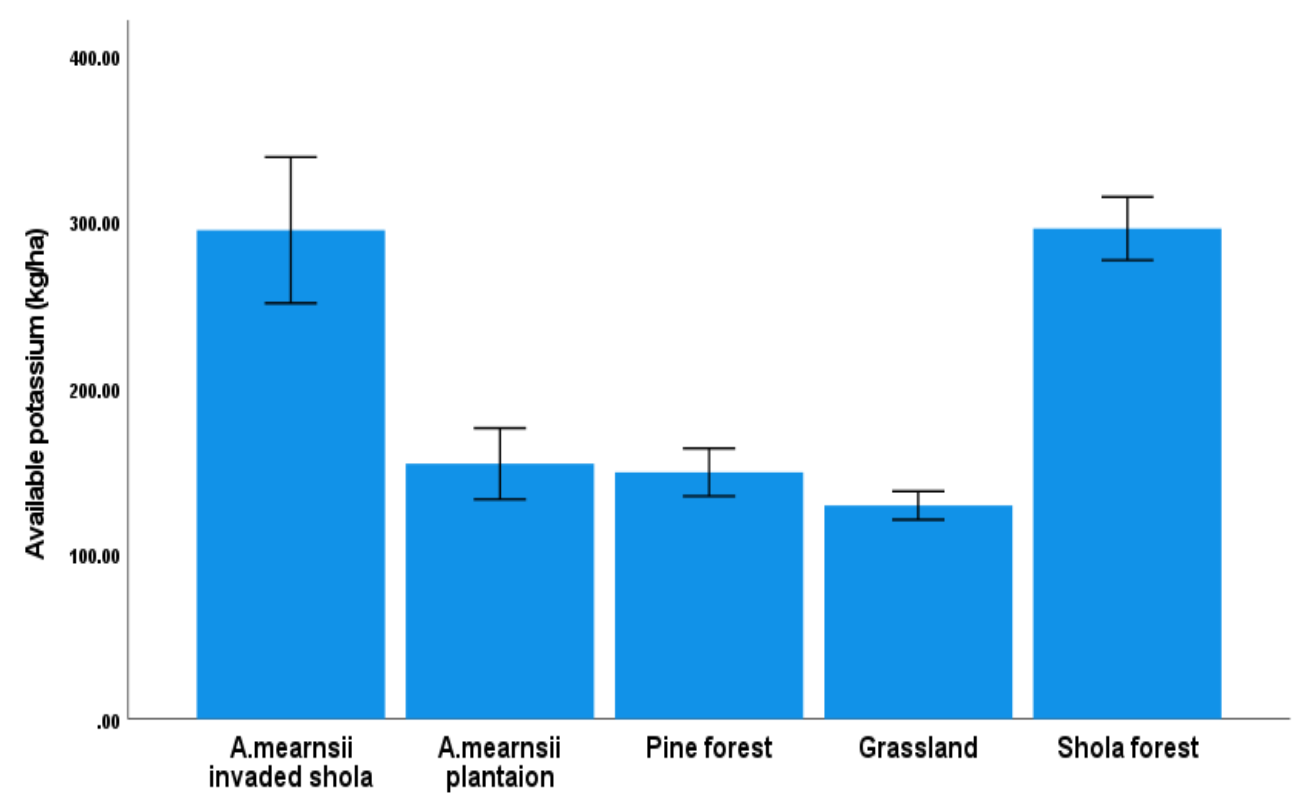

Figure 5. A. Mearnsii invasion on available potassium $\left(\mathrm{kg} \mathrm{ha}^{-1}\right)$ in Poombarai range

The effects of $A$. mearnsii invasion on soil exchangeable calcium in the Poombarai range at $0-30 \mathrm{~cm}$ is presented in (Table 1). The exchangeable calcium varied from 0.3 to $0.62 \mathrm{meq} / 100 \mathrm{~g}$. The exchangeable calcium was highest under $A$. mearnsii invaded shola forest $(3.8 \mathrm{meq} / 100 \mathrm{~g})$ at $0-30 \mathrm{~cm}$ depth. This was followed by shola forest $(3.6 \mathrm{meq} / 100 \mathrm{~g})$ and grassland $(3.6 \mathrm{meq} / 100 \mathrm{~g})$. A. mearnsii plantation registered the lowest exchangeable calcium $(2.0 \mathrm{meq} / 100 \mathrm{~g})$ at $0-30 \mathrm{~cm}$ depth. Exchangeable calcium availability was highest $(\mathrm{p}<0.001 \%)$ under shola forest and was on par with $A$. mearnsii invaded shola forest at $30-50 \mathrm{~cm}$ (Table 2).

The perusal of data on exchangeable magnesium in the different study plots revealed that, at $0-30 \mathrm{~cm}$ depth, A. mearnsii invaded shola forest recorded the highest exchangeable magnesium $(5.2 \mathrm{meq} / 100 \mathrm{~g})$. This was found to be on par with the shola forest $(5.2 \mathrm{meq} / 100 \mathrm{~g})$. The exchangeable magnesium showed a decreasing trend with soil depth in all the study plots (Table 1). At $30-50 \mathrm{~cm}$ and $50-80 \mathrm{~cm}$, A. mearnsii invaded shola forest registered higher exchangeable magnesium $(\mathrm{p}<0.001)$ and was on par with shola forest (Tables 2 and 3). 


\section{Micronutrients availability}

Among the different study plots, shola forests recorded the highest DTPA-Cu (5.6 ppm), DTPA-Zn (1.2 ppm), DTPA-Mn (2.6 ppm), DTPA-Fe (20.1 ppm) at 0-30 cm depth (Table 1; Fig. 6) and was followed by A. mearnsii invaded shola forest (3.0, 1.0, 2.4 and $10.4 \mathrm{ppm})$, and grassland $(2.8,0.8,0.4$ and $6.4 \mathrm{ppm})$. The lowest DTPA-Cu (0.6 ppm), DTPA-Fe (5.9 ppm), DTPA-Mn (0.2 ppm) and DTPA-Zn (0.3 ppm) was recorded in pine forest at 0 to $30 \mathrm{~cm}$ depth. At $30-50$ and $50-80 \mathrm{~cm}$, shola forest registered the highest DTPA-Cu, DTPA-Mn and DTPA-Fe $(\mathrm{p}<0.001)$. Pine forest recorded the lowest availability of micronutrients with respect to copper, iron manganese and zinc (Tables 2 and 3). DTPA-Cu, DTPA-Zn and DTPA-Fe availability was highest $(\mathrm{p}<0.001)$ under shola forest at $80-100 \mathrm{~cm}($ Table 4$)$.

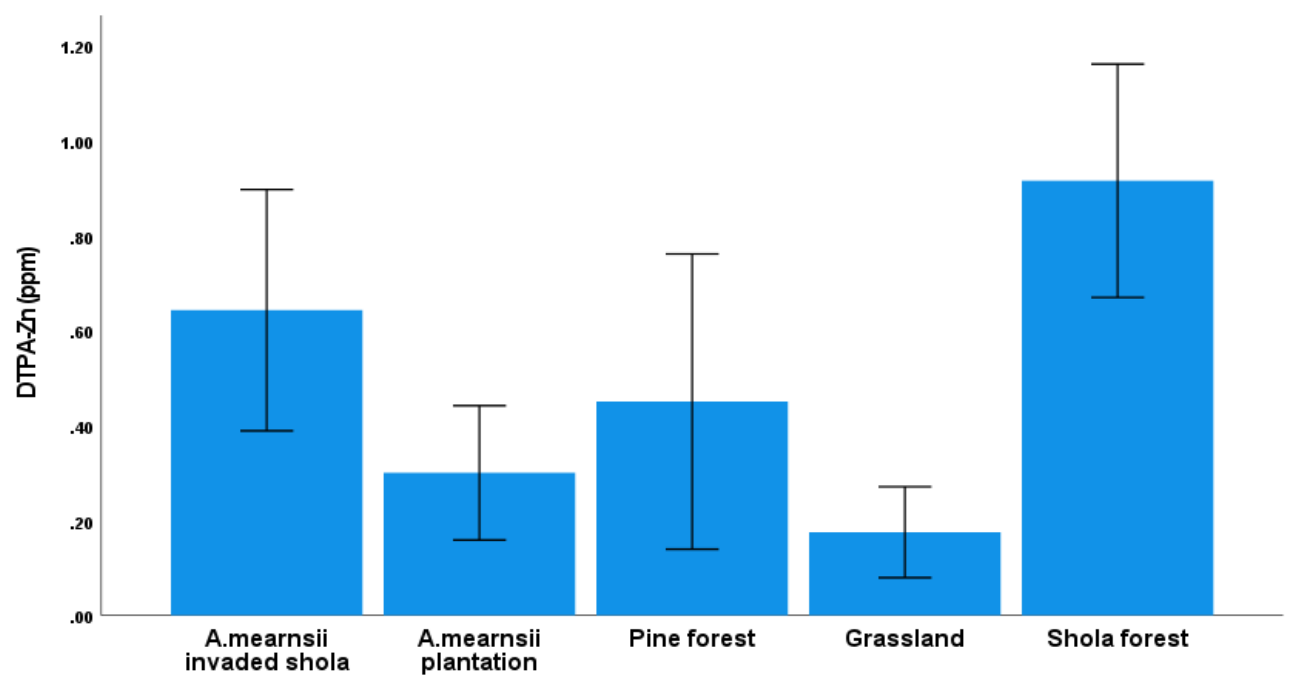

Figure 6. A. mearnsii invasion on DTPA-Zn (ppm) in Poombarai range

\section{Discussion}

Introduced plants affect soil nutrient dynamics by differing from native species in tissue chemistry, plant morphology and phenology (Ehrenfeld, 2003). Soil properties such as $\mathrm{pH}$ and the amount of base cations available in the soil differ greatly under trees of different species and when invading trees are compared with native vegetation (Dijkstra et al., 2001). The present study revealed higher $\mathrm{pH}$ under A. mearnsii invaded shola forest. Similar results were reported for L. camara infested soils by Fan et al. (2010) and Olusegun and Perrett (2011). High soil pH accelerated litter decomposition and thus plays a crucial role in regulating nutrient availability. Due to the high soil cation exchange process, soil cations are available in the root rhizosphere of the invasive species compared to native species resulting in high soil $\mathrm{pH}$ in the invaded sites. (Simba et al., 2013; Debnath et al., 2018). However, both increases and decreases in $\mathrm{pH}$ following plant invasion have been equally reported in the literature (Ehrenfeld, 2003).

Soil organic carbon was highest under A. mearnsii invaded shola forest. The results are concurrent with many other studies (Picone et al., 2003; Cheng et al., 2013). The combined effect of low temperature and high rainfall in shola forests restricts biochemical decomposition of organic residues in these soils and thus help maintain 
high organic carbon percentage, which in turn becomes responsible for the high cation exchange capacity and base saturation of these soils (Balagopalan, 1993). However, Debnath et al. (2018) reported equal amount of soil organic carbon in both invaded and natural sites. Available nitrogen, exchangeable calcium and magnesium were highest under $A$. mearnsii invaded shola forest. The increase in available nitrogen and exchangeable calcium and magnesium in A. mearnsii invaded sites could be due to decrease in nutrient sequestration following native shola species displacement. $A$. mearnsii drops a lot of litter beneath it and this probably account for the elevated nitrogen levels when the litter decays. These findings are consistent with the findings by Ehrenfeld (2003), who reported an increase in soil nitrate following Lantana camara invasion. Jovanovic et al. (2009) also reported an increase in nitrogen where A. saligna invaded Sand Plain Fynbos. Dusanka et al. (2021) also reported that soil in mixed plots (those populated with Amorpha fruticosa, Fraxinus pennsylvanica and Acer negundo) contained much higher levels of nitrifying bacteria (NB), organic matter $(\mathrm{Om})$, nitrogen $(\mathrm{N})$, and carbon $(\mathrm{C})$ as well as lower carbon to nitrogen ratio $(\mathrm{C}: \mathrm{N})$ levels, compared to single species invaded plots and control plots.

In our study, A. mearnsii plantation recorded the highest available phosphorus and was on par with shola forest. Musil and Midgley (1990) found similar results on Sand Plain Fynbos. Acacia saligna infestation doubled the average concentration of calcium, magnesium, potassium, total nitrogen, manganese, boron and zinc. Burnt plots showed a significant increase for soil $\mathrm{pH}$, calcium, manganese and available phosphorus.

Our findings revealed that, available potassium was highest under A. mearnsii invaded shola forest. The high soil cation exchange process might have resulted in increased soil cations availability in the root rhizosphere of the invasive species compared to native species and our results was supported by earlier findings of Simba et al. (2013), Ruwanza and Shackleton (2016) and Debnath et al. (2018). Similarly, a study on long-term invasive occupation by Acacia longifolia significantly altered soil properties, with increased levels of organic $\mathrm{C}$, total $\mathrm{N}$, and exchangeable cations (Ehrenfeld, 2003 and Fan et al. 2010). In the present study, the availability of micronutrients viz, DTPA-Cu, Zn Mn and Fe were higher under shola forest compared to other study plots.

\section{Conclusion}

For proper management of the environment and utilization of resources, studies on the properties of soil in invaded forest ecosystems are important. Without adequate knowledge of the dynamic interaction between soil, climate and forest management we cannot develop a proper soil management system. Soil properties such as $\mathrm{pH}$, available nitrogen, available phosphorus, available potassium, exchangeable calcium and magnesium in the soil varied significantly under A. mearnsii invaded shola forests compared with native vegetation. Baseline data generated in the present study can be utilized by the State Forest Department for undertaking appropriate decision making in the eco-restoration of areas invaded by A. mearnsii. From a management point of view removal of $A$. mearnsii for restoration purposes, could reduce the effect of the invasive species on the soil properties. Therefore, appropriate methods for the management of $A$. mearnsii are necessary to circumvent potential threats to native biodiversity. 
Acknowledgements. We are thankful to the Director General, Indian Council of Forestry Research and Education, Dehradun for providing financial support to undertake the project work.

\section{REFERENCES}

[1] Balagopalan, M., Jose, A. I. (1993): A comparative study on the properties of soils in relation to the vegetational types. - Journal of Tropical Agriculture 31: 167-173.

[2] Bray, R. H., Kurtz, L. T. (1945): Determination of total, organic and available forms of phosphorus in soils. - Soil Science 59: 39-45.

[3] Cheng, K. L., Bray, R. H. (1951): Determination of calcium and magnesium in soil and plant material. - Soil Science 72:449-458.

[4] Cheng, C. H., Chen, Y. S., Huang., C.-R., Chiou, C.-C., Lin, D., Oleg, V. M. (2013): Effects of repeated fires on ecosystem $\mathrm{C}$ and $\mathrm{N}$ stocks along a fire induced forest/grassland gradient. - Journal of Geophysical Research 118: 215-25.

[5] Debnath, A., Paul, R., Debnath, B. (2018): Effects of the invasive shrub, Chromolaena odorata, on soil properties in the Atharamur a forest ecosystem: Indian Himalayan state of Tripura, Northeast India. - Vegetos 31(2):77-90. DOI: 10.5958/2229-4473.2018.00059.9.

[6] Dijkstra, F. A. (2001): The effect of organic acids on base cation leaching from the forest floor under six North American tree species. - European Journal of Soil Science 52: 205-214.

[7] Dusanka, V., Gianalberto, L., Stanko, M., Dubravka, M. (2021): The impact of multiple species invasion on soil and plant communities increases with invader diversity. - Biorxiv. DOI: 10.1101/2021.04.30.442106

[8] Ehrenfeld, J. G. (2003): Effects of exotic plant invasions on soil nutrient cycling processes. - Ecosystems 6: 503-523.

[9] Fan, L., Chen, Y., Yuan, J., Yang, Z. (2010): The effect of Lantana camara Linn. invasion on soil chemical and microbiological properties and plant biomass accumulation in southern China. - Geoderma 154: 370-378.

[10] Galatowitsch, S., Richardson, D. M. (2005): Riparian scrub recovery after clearing of invasive alien trees in headwater streams of the Western Cape, South Africa. - Biological Conservation 122: 509-521.

[11] Haubensaket, K. A., D’Antonio, C. M., Alexander, J. (2004): Effects of nitrogen-fixing shrubs in Washington and coastal California. - Weed Technology 18: 1475-1479.

[12] Hawkes, C. V., Wren, I. F., Herman, D. J., Firestone, M. K. (2005): Plant invasion alters nitrogen cycling by modifying the soil nitrifying community. - Ecology Letters 8: 976-985.

[13] Jackson, M. L. (1973): Soil and Plant Analysis. - Prentice Hall of India Private Limited, New Delhi.

[14] Jovanovic, N. Z., Israel, S., Tredoux, G., Soltau, L., Le Maitre, D., Rusinga, F., Rozanov, A., Van der Merwe, N. (2009): Nitrogen dynamics in land cleared of alien vegetation (Acacia saligna) and impacts on groundwater at Riverlands Nature Reserve (Western Cape, South Africa). - Water Sa 35(1): 37-44.

[15] Kumar, S. (1993): Survey and mapping of shola forests and grasslands in the upper Nilgiri plateau and assessment of human utilization of the vegetation. - Report submitted to World Wide Fund for Nature India. WWF, Gland.

[16] Lindsay, W. L., Norvell, W. A. (1978): Development of DTPA soil test for zinc, iron, manganese and copper. - Soil Science Society of American Journal 42: 421-428.

[17] Mathew, K. M., Blasco, F., Ignacimuthu, S. (1975): Biological changes at Kodaikanal, 1949-1974. - Tropical Ecology 16: 147-162.

[18] Mitchell, N. (1972): The Indian hill-station: Kodaikanal. - University of Chicago Department of Geography Research, Paper No.141, University of Chicago, Chicago.

[19] Moyo, H. P. M., Fatunbi, A. O. (2010): Utilitarian perspective of the invasion of some South African biomes by A. mearnsii. - Global Journal of Environmental Research 4(1): 6-17. 
[20] Musil, C. F. (1993): Effects of invasive Australian acacias on the regeneration, growth and nutrient chemistry of South African lowland Fynbos. - Journal of Applied Ecology 30: 361-372.

[21] Myers, N., Mittermeier, R. A., Mittermeier, C. G., Da Fonseca, G. A. B., Kent, J. (2000): Biodiversity hotspots for conservation priorities. - Nature 403: 853-858.

[22] Olsen, S. R., Cole, C. V., Watanabe, F. S., Dean, A. L. (1954): Estimation of available phosphorus in soils by extraction with sodium bicarbonate. - Circular No.: 939, USDFA, US Govt. Printing Office, Washington, DC.

[23] Olusegun, O. O., Perrett, C. (2011): Lantana camara L. (Verbenaceae) invasion effects on soil physico-chemical properties. - Biology and Fertility of Soils 47: 349-355.

[24] Pandey, S. K., Singh, H., Singh, J. S. (2014): Effect of environmental conditions on decomposition in eight woody species of a dry tropical forest. - Plant Biosystem 148: 410418. https://doi.org/10.1080/11263504.2013.772923.

[25] Picone, L. I., Quaglia, G., Garcia, F. O., Laterra, P. (2003): Biological and chemical response of a grassland soil to burning. - Journal of Range Management 56(3): 291-97.

[26] Reddy, T. Y., Reddy, G. H. S. (2010): Principles of Agronomy. - Kalyani Publishers, New Delhi.

[27] Ruwanza, S., Shackleton, C. (2016): Effects of the invasive shrub, Lantana camara, on soil properties in the Eastern Cape, South Africa. - Weed Biology and Management 16: 67-79. https://doi.org/10.1111/ wbm.12094.

[28] Ruwanza, S., Gaertner, M., Richardson, D. M., Esler, K. J. (2013): Soil water repellency in riparian systems invaded by Eucalyptus camaldulensis: a restoration perspective from the Western Cape Province, South Africa. - Geoderma 200-201: 9-17. https://doi.org/10.1016/j.-Geoderma 2013.01.017.

[29] Simba, Y. R., Kamweya, A. M., Mwangi, P. N., Ochora, J. M. (2013): Impact of the invasive shrub Lantana camara L. on soil properties in Naibory National Park, Kenya. International Journal of Biodiversity Conservation 5(12): 803-809. https://doi.org/10.5895/IJBC2013.0623.

[30] Stanford, G., English, L. (1949): Use of flame photometer in rapid soil test for K and Ca. Agronomy Journal.41: 446-447.

[31] Subbiah, B. V., Asija, G. L. (1956): A rapid procedure for the estimation of available nitrogen in soils. - Current Science 25: 259-260.

[32] Tererai, F., Gaertner, M., Jacobs, S. M., Richardson, D. M. (2015): Eucalyptus camaldulensis invasion in riparian zones reveals few significant effects on soil physicochemical properties. - River Res Appl 31: 590-601. https://doi.org/10.1002/rra.2762.

[33] Vardien, W., Richardson, D. M., Foxcroft, L. C., Thompson, G. D., Wilson, J. R. U. (2012): Invasion dynamics of Lantana camara L. (sensu lato) in South Africa. - African Journal of Botany 81: 81-94.

[34] Walkley, A., Black, C. A. (1934): An examination of the Degtjareff method for determining soil organic matter and a proposed modification of the chromic acid titration method. - Soil Science 40: 233- 243.

\section{APPENDIX}

Appendix 1. Details of soil sample collection in Kodaikanal (Poombarai Range)

\begin{tabular}{|c|c|c|c|c|}
\hline S. No. & Soil depth & Location & GPS & Forest type \\
\hline 1 & $0-30 \mathrm{~cm}$ & \multirow{3}{*}{$\begin{array}{l}\text { Near Kundar Falls } \\
\text { (Kodaikanal) }\end{array}$} & \multirow{3}{*}{ 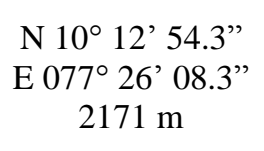 } & \multirow{3}{*}{ Pine forest plantation } \\
\hline 2 & $30-50 \mathrm{~cm}$ & & & \\
\hline 3 & $50-80 \mathrm{~cm}$ & & & \\
\hline
\end{tabular}




\begin{tabular}{|c|c|c|c|c|}
\hline 4 & $80-100 \mathrm{~cm}$ & & & \\
\hline 5 & $0-30 \mathrm{~cm}$ & \multirow{4}{*}{$\begin{array}{l}\text { Near Kundar Falls } \\
\text { (Kodaikanal) }\end{array}$} & \multirow{4}{*}{$\begin{array}{l}\text { N } 10^{\circ} 21^{\prime} 58.2^{\prime} \\
\text { E } 077^{\circ} 43^{\prime} 62.7^{\prime} \\
2171 \mathrm{~m}\end{array}$} & \multirow{4}{*}{ Pine forest plantation } \\
\hline 6 & $30-50 \mathrm{~cm}$ & & & \\
\hline 7 & $50-80 \mathrm{~cm}$ & & & \\
\hline 8 & $80-100 \mathrm{~cm}$ & & & \\
\hline 9 & $0-30 \mathrm{~cm}$ & \multirow{4}{*}{ Kundar Falls (Kodaikanal) } & \multirow{4}{*}{$\begin{array}{l}\mathrm{N} 10^{\circ} 13^{\prime} 02.7^{\prime \prime} \\
\mathrm{E} 077^{\circ} 25^{\prime} 33.3^{\prime \prime} \\
2185 \mathrm{~m}\end{array}$} & \multirow{4}{*}{ Grass land } \\
\hline 10 & $30-50 \mathrm{~cm}$ & & & \\
\hline 11 & $50-80 \mathrm{~cm}$ & & & \\
\hline 12 & $80-100 \mathrm{~cm}$ & & & \\
\hline 13 & $0-30 \mathrm{~cm}$ & \multirow{4}{*}{ Kundar Falls (Kodaikanal) } & \multirow{4}{*}{$\begin{array}{l}\mathrm{N} 10^{\circ} 21^{\prime} 72.0^{\prime \prime} \\
\text { E } 077^{\circ} 42^{\prime} 67.1^{\prime \prime} \\
2131 \mathrm{~m}\end{array}$} & \multirow{4}{*}{ Grass land } \\
\hline 14 & $30-50 \mathrm{~cm}$ & & & \\
\hline 15 & $50-80 \mathrm{~cm}$ & & & \\
\hline 16 & $80-100$ & & & \\
\hline 17 & $0-30 \mathrm{~cm}$ & \multirow{4}{*}{ Krishnan Kovil (Kodaikanal) } & \multirow{4}{*}{ 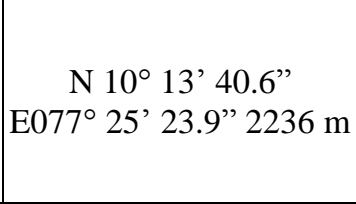 } & \multirow{4}{*}{$\begin{array}{l}\text { Acacia mearnsii } \\
\text { plantation }\end{array}$} \\
\hline 18 & $30-50 \mathrm{~cm}$ & & & \\
\hline 19 & $50-80 \mathrm{~cm}$ & & & \\
\hline 20 & $80-100 \mathrm{~cm}$ & & & \\
\hline 21 & $0-30 \mathrm{~cm}$ & \multirow{4}{*}{ Krishnan Kovil (Kodaikanal) } & \multirow{4}{*}{$\begin{array}{c}\mathrm{N} 10^{\circ} 22^{\prime} 82.4 ” \\
\mathrm{E} 077^{\circ} 42^{\prime} \\
32.3^{\prime \prime}\end{array}$} & \multirow{4}{*}{$\begin{array}{l}\text { Acacia mearnsii } \\
\text { plantation }\end{array}$} \\
\hline 22 & $30-50 \mathrm{~cm}$ & & & \\
\hline 23 & $50-80 \mathrm{~cm}$ & & & \\
\hline 24 & $80-100 \mathrm{~cm}$ & & & \\
\hline 25 & $0-30 \mathrm{~cm}$ & \multirow{4}{*}{$\begin{array}{l}\text { FDA thottam (TNFD) inside } \\
\text { the Krishnan kovil } \\
\text { (Kodaikanal) }\end{array}$} & \multirow{4}{*}{ 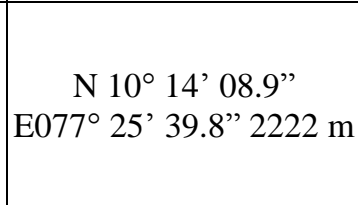 } & \multirow{4}{*}{$\begin{array}{l}\text { Acacia mearnsii } \\
\text { invaded grass land }\end{array}$} \\
\hline 26 & $30-50 \mathrm{~cm}$ & & & \\
\hline 27 & $50-80 \mathrm{~cm}$ & & & \\
\hline 28 & $80-100 \mathrm{~cm}$ & & & \\
\hline 29 & $0-30 \mathrm{~cm}$ & \multirow{4}{*}{$\begin{array}{c}\text { FDA thottam (TNFD) inside } \\
\text { the Krishnan kovil } \\
\text { (Kodaikanal) }\end{array}$} & \multirow{4}{*}{$\begin{array}{c}\mathrm{N} 10^{\circ} 23,53.7 ” \\
\mathrm{E} 077^{\circ} 42^{\prime}, 78.0^{\prime \prime} 2213 \mathrm{~m}\end{array}$} & \multirow{4}{*}{$\begin{array}{l}\text { Acacia mearnsii } \\
\text { invaded grass land }\end{array}$} \\
\hline 30 & $30-50 \mathrm{~cm}$ & & & \\
\hline 31 & $50-80 \mathrm{~cm}$ & & & \\
\hline 32 & $80-100 \mathrm{~cm}$ & & & \\
\hline 33 & $0-30 \mathrm{~cm}$ & \multirow{4}{*}{$\begin{array}{c}\text { Near Water flow after Krishnan } \\
\text { temple (Kodaikanal) }\end{array}$} & \multirow{4}{*}{$\begin{array}{c}\mathrm{N} 10^{\circ} 14^{\prime} 23.3^{\prime \prime} \\
\mathrm{E} 077^{\circ} 25^{\prime} \\
20.1 ” 2193 \mathrm{~m}\end{array}$} & \multirow{4}{*}{ Shola forest } \\
\hline 34 & $30-50 \mathrm{~cm}$ & & & \\
\hline 35 & $50-80 \mathrm{~cm}$ & & & \\
\hline 36 & $80-100 \mathrm{~cm}$ & & & \\
\hline 37 & $0-30 \mathrm{~cm}$ & & & \\
\hline 38 & $30-50 \mathrm{~cm}$ & Near Water flow after Krishnan & $\mathrm{N} 10^{\circ} 24^{\prime} 02.4^{\prime \prime}$ & Sholo foroct \\
\hline 39 & $50-80 \mathrm{~cm}$ & temple (Kodaikanal) & E077 42’ 22.9” $2201 \mathrm{~m}$ & snora iorest \\
\hline 40 & $80-100 \mathrm{~cm}$ & & & \\
\hline 41 & $0-30 \mathrm{~cm}$ & & & \\
\hline 42 & $30-50 \mathrm{~cm}$ & Near Mahalakshmi kovil & $\mathrm{N} 10^{\circ} 14^{\prime} 56.5^{\prime \prime}$ & Acacia mearnsii \\
\hline 43 & $50-80 \mathrm{~cm}$ & (Kodaikanal) & E077 24’ 53.5”2194 m & invaded shola forest \\
\hline 44 & $80-100 \mathrm{~cm}$ & & & \\
\hline 45 & $0-30 \mathrm{~cm}$ & & & \\
\hline 46 & $30-50 \mathrm{~cm}$ & Near Mahalakshmi kovil & $\mathrm{N} 10^{\circ} 24^{\prime} 86.8^{\prime \prime}$ & Acacia mearnsii \\
\hline 47 & $50-80 \mathrm{~cm}$ & (Kodaikanal) & $\mathrm{E} 077^{\circ} 41^{\prime} 46.0^{\prime \prime} 2103 \mathrm{~m}$ & invaded shola forest \\
\hline 48 & $80-100 \mathrm{~cm}$ & & & \\
\hline
\end{tabular}

